\title{
Ploidy Level in the True Slime Mould Didymium nigripes
}

\author{
By SYLVIA KERR \\ Department of Zoology, University of Minnesota, \\ Minneapolis, Minnesota, 55455, U.S.A.
}

(Accepted for publication I March 1968)

\begin{abstract}
SUMMARY
Estimation of chromosome numbers in approximately Iooo nuclei of amoebae and plasmodia of the true slime mould, Didymium nigripes, has shown that in the $\mathrm{C} 6$ and $\mathrm{s} 3$ strains the ploidy level of amoebae and plasmodia is the same. Nuclei with differing chromosome numbers were found in the populations studied. The data collected here are consistent with the hypothesis that as a culture ages during serial subculture of one stage of the life-cycle, nuclei with higher than normal chromosome numbers accumulate.
\end{abstract}

\section{INTRODUCTION}

During the course of its life-cycle the true slime mould Didymium nigripes exhibits several strikingly different morphological phases. In routine laboratory culture spores plated on agar in the presence of a food source, Aerobacter aerogenes, germinate to form uninucleate amoebae. After several days of growth and division members of the amoeboid population differentiate to form multinucleate coenocytic plasmodia. If subjected to conditions of starvation a plasmodium differentiates to form fruiting bodies. Cleavage within the sporangium then leads to production of uninucleate spores, thus completing the life-cycle. Heterothallism or a mating-type incompatibility system is absent in this isolate. Fusion of amoebae to form a 'plasmodial precursor cell' and meiosis during sporulation have not been confirmed by either genetic or cytological means.

Recent studies of mitosis in Didymium nigripes (S. Kerr, 1967) have shown that mitotic behaviour and the time course of division differ in the amoeboid and plasmodial stages of the life-cycle. The work reported here was undertaken to determine whether or not these differences in behaviour could be correlated with a change in ploidy level, which historically has been implicated as the initiating step in plasmodial differentiation. Accurate chromosome counts for the myxomycetes are nearly impossible to obtain because the chromosomes of this group of organisms are extremely small and fairly numerous. Nevertheless, estimation of ploidy level, based on chromosome counts, is feasible. This technique has been used previously by Ross (I966) to determine approximate chromosome number in three other species of slime moulds: Physarum polycephalum, $P$. flavicomum and Badhamia curtisii.

In the present study ploidy level was estimated in amoebae and plasmodia of two clonally derived strains of a single isolate of Didymium nigripes grown on living bacteria. One strain was a wild-type clone, c6 (N. Kerr, 1965), and the other was a minute mutant, s3 (N. Kerr, I967), known to undergo plasmodium formation without 
plasmogamy. Chromosome numbers were also estimated in four strains (also derived from the same isolate) of 'axenically grown' amoebae which had been subcultured for varying lengths of time on formalin-killed bacteria.

\section{METHODS}

Amoebae were grown as described elsewhere (N. Kerr, 1960, 1961, 1963) and in mid-log phase of growth were harvested in Schaudinn fixative $(66 \mathrm{ml}$. water saturated with $\mathrm{HgCl}_{2}+33 \mathrm{ml} .95 \% \mathrm{EtOH}+\mathrm{I} \mathrm{ml}$. glacial acetic acid) and stored for $\mathrm{I} 2 \mathrm{hr}$. The tube was then centrifuged, the fixative decanted and $65 \%$ acetic acid added. After 30 min. a drop of cell suspension was placed on a slide, $2 \%$ aceto-orcein was added, the coverslip was pressed slightly to remove excess liquid and the preparations were sealed with paraffin. In all cases the time elapsed between inoculation of the medium with spores and harvesting of the amoebae was noted; this elapsed time has been designated as the 'age' of the culture.

Young plasmodia develop after 2 days (c6) and 3 days $\left(\mathrm{s}_{3}\right)$ of growth on $0.0 \mathrm{I}$ Mphosphate buffered agar, $\mathrm{pH} 6 \cdot 5$, (S. Kerr, 1967) in cultures seeded with spores from a single sporangium. Amoebae and plasmodia from these cultures were harvested and incubated as described above and slides were prepared with glycerine as the mounting medium. Microplasmodia were obtained by scraping pieces of plasmodia which had grown several days on agar, suspending them in phosphate buffer and breaking them up with a Vortex junior mixer. The resulting microplasmodia were incubated for I $2 \mathrm{hr}$ on phosphate-buffered agar to allow asynchrony of mitosis to develop and then harvested and treated as above. Microplasmodia formed in this manner grew normally and completely covered an agar plate in 2 days; the technique simply facilitated obtaining small pieces of plasmodium which were easy to handle and increased the probability of finding dividing nuclei.

All observations were made by phase-contrast microscopy using a Zeiss GFL microscope with a $\times 100$ oil apochromat objective. Duplicate counts of chromosomes either in prometaphase or in metaphase were taken on nuclei where the disposition of chromosomes was favourable. Counts from a small number of nuclei were discarded because the two counts were not within $5 \%$ of each other. Photographs through several focal levels of representative nuclei were taken with a $35 \mathrm{~mm}$. camera on Kodak high-contrast copy film. Enlargements of these photographs were used for a second estimate of chromosome number (Pl. I.) Although chromosomes can be readily distinguished from bacteria enclosed in food vacuoles after a little practice, chromosome counts were made only when the presence of a spindle could be confirmed. The presence of a nuclear membrane was used to distinguish plasmodial nuclei from those of amoebae.

\section{RESULTS}

Eight populations of amoebae were surveyed: c6 wild-type cells grown on SS agar (N. Kerr, 1967) which permits plasmodial differentiation and on GPY-B agar (N. Kerr, I960) which inhibits such differentiation; $s 3$ cells grown on SS and GPY-B agar, both of which permit differentiation of this strain; and four strains of cells grown in 'axenic' culture (IO I wild-type, $\mathrm{c} 6$ wild-type, $\mathrm{s} 3$ and A 20 ; see N. Kerr, 1963). Chromosome number estimates were made for 100 metaphase cells of each population. 
Young plasmodia containing $\mathrm{I}-4$ nuclei were examined in $\mathrm{c} 6$ and $\mathrm{s} 3$ populations grown from spores on SS agar. Nuclei of older plasmodia were examined in microplasmodia derived from $\mathrm{c} 6$ and $\mathrm{s} 3$ plasmodia of several ages.

Pooled data from all c 6 and $s 3$ amoebae (60o cells) are shown in Fig. I. On the basis of these counts, one ploidy level (class A) contains between $3 \mathrm{I}$ and 38 chromosomes (Pl. I, fig. I, 8, 9). A second ploidy level(class C) exists which contains twice this number or about 6I-76 chromosomes (PI. I, fig. 2, 3). Some nuclei were found which could not be placed in either of the above categories. For purposes of the statistical analysis numbers from 39 to 60 ( 172 nuclei) were grouped in class B and numbers over I00 (I 7 nuclei)

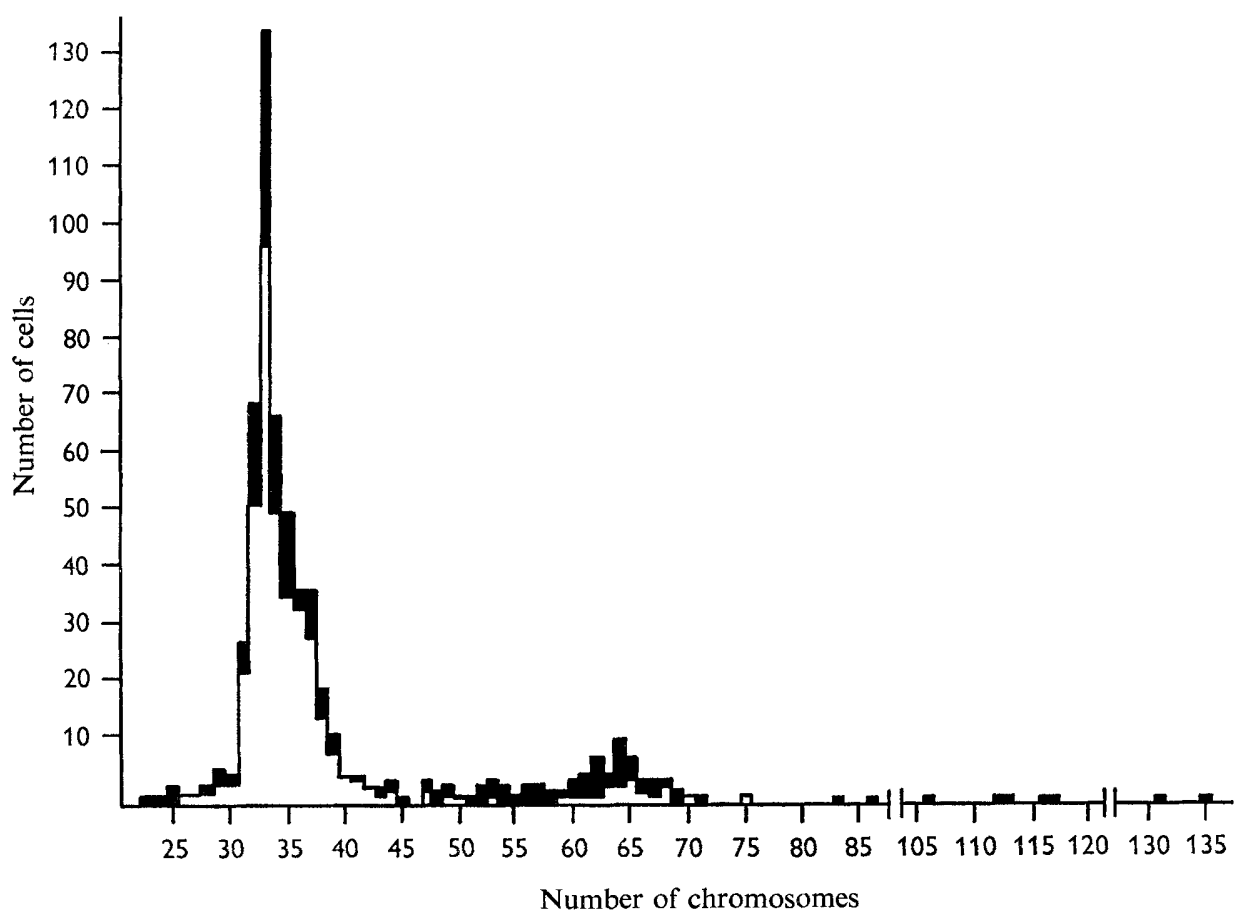

Fig. 1. Chromosome number distribution in c6 and s3 amoebae. $\mathbf{n}$, Axenically grown; $\square$, bacterial-grown.

Table I. Comparison of ploidy level in amoebae and plasmodia

Strain and culture conditions C6 amoebae SS grown; $45 \mathrm{hr}$ C6 amoebae GPY-B grown; $65 \mathrm{hr}$ c6 young plasmodia SS grown; $45 \mathrm{hr}$ c6 microplasmodia various ages (30-200 days)

s3 amoebae SS grown; $45 \mathrm{hr}$ s3 amoebae GPY-B grown; $65 \mathrm{hr}$ s3 young plasmodia SS grown; $96 \mathrm{hr}$ s3 microplasmodia 60 days

\begin{tabular}{|c|c|c|c|c|c|c|}
\hline \multicolumn{6}{|c|}{ Approximate number of chromosomes } & \multirow{2}{*}{$\begin{array}{c}\text { Total } \\
\text { nuclei } \\
\text { examined }\end{array}$} \\
\hline$<3 I$ & $3 I-38$ & $39-60$ & $6 I-76$ & $77-100$ & $>100$ & \\
\hline I & 94 & 2 & 3 & 0 & 0 & 100 \\
\hline 6 & 71 & 20 & 2 & I & 0 & 100 \\
\hline I & 43 & 5 & 2 & 0 & 0 & $5 \mathrm{I}$ \\
\hline I & 24 & 4 & 4 & 2 & 0 & 35 \\
\hline 0 & 93 & I & 6 & 0 & o & 100 \\
\hline 3 & 81 & 8 & 8 & 0 & 0 & 100 \\
\hline 0 & 46 & I & 3 & 0 & 0 & 50 \\
\hline 0 & 24 & 0 & 2 & 0 & 0 & 26 \\
\hline
\end{tabular}


were placed in Class D (PI. I, fig. 4, 5). Fewer than 31 chromosomes were counted in 30 cells. The distribution of nuclei by chromosome number classes is shown in Tables I and 3 .

If class $\mathrm{A}$ represents the haploid chromosome number and class $\mathrm{C}$ represents the diploid, a comparison of these two classes in amoebae and plasmodia would tell whether karyogamy precedes plasmodial differentiation. Visual inspection of Table I shows that the majority of nuclei of both amoebae and plasmodia belong to class $\mathrm{A}$. Thus in $\mathrm{c} 6$ and $\mathrm{s}_{3}$ karyogamy can not be a prerequisite for plasmodium formation.

The presence in the populations of nuclei with chromosome numbers which do not fall in class A remains unexplained. Some variation in numbers of nuclei which belong to classes B, C and D is apparent when different populations are compared. Data shown in Table I were analysed by means of $\chi^{2}$ contingency tables. When the distribution of all chromosome number classes for $\mathrm{c} 6$ and $\mathrm{s} 3$ bacterially grown populations was analysed, $s_{3}$ showed no significant difference in total distribution among classes $(P=0.33)$ whereas the difference in distribution in $\mathbf{C} 6$ was significant $(P<0.001)$.

Table 2. $\chi^{2}$ evaluation of class $A$ : class $C$ distributions

\section{Populations compared}

s3 amoebae, young plasmodia and microplasmodia c6 amoebae, young plasmodia and microplasmodia c6 amoebae and young plasmodia

Level of
significance
0.89
0.08
0.83

Table 3. Comparison of ploidy level in axenic amoebae

\begin{tabular}{|c|c|c|c|c|c|c|c|}
\hline \multirow[b]{2}{*}{ Strain and age } & \multicolumn{6}{|c|}{ Approximate number of chromosomes } & \multirow{2}{*}{$\begin{array}{c}\text { Total } \\
\text { nuclei } \\
\text { examined }\end{array}$} \\
\hline & $<31$ & $3 I-38$ & $39-60$ & $6 I-76$ & $77-100$ & $>100$ & \\
\hline c6; 9 months & 2 & 66 & 4 & 24 & 0 & 4 & 100 \\
\hline $\mathrm{s}_{3} ; 2$ years & 9 & 42 & 34 & IO & 2 & 3 & 100 \\
\hline A $20 ; 3$ years & 7 & 20 & $4 I$ & 21 & 7 & 4 & 100 \\
\hline IOI ; $6 \frac{1}{2}$ years & 0 & 8 & 52 & 31 & 3 & 6 & 100 \\
\hline
\end{tabular}

In an attempt to determine the source of variability of total distribution, the data in Table I were regrouped and analysed as above. Class A (3I-38):class C (6I-76) distributions were compared to determine whether the proportions of cells in these classes differed in the various populations. The results of this analysis are presented in Table 2. Note that, as above, $s 3$ populations showed no significant difference in distribution. When $\mathrm{C} 6$ amoebae, young plasmodia and microplasmodia were compared the difference was nearly significant, whereas if only amoebae and young plasmodia were compared the populations showed similar distributions. Thus the $\mathrm{c} 6$ microplasmodia represent one major source of variability.

One possible explanation for this difference in ploidy level is that the $\mathrm{c} 6$ microplasmodia were derived from plasmodia which had 'aged' up to 200 days whereas $\mathrm{s} 3$ microplasmodia were derived from fairly young plasmodia. In an attempt to test the hypothesis that chromosome number may vary with ageing, axenically grown amoebae which had been serially subcultured for varying lengths of time were examined (see Table 3). Comparison of the numbers of nuclei with $3 \mathrm{I}-38$ chromosomes in these populations shows that the youngest culture (c6 axenic) possessed a majority of nuclei with this number of chromosomes but that as the age of these cultures increased, 
the proportion of cells containing chromosome numbers outside the $3 \mathrm{I}-38$ class also increased. A parallel trend can be noted when bacterial-grown amoebae are compared after 45 and $65 \mathrm{hr}$ of growth.

\section{Ancestry of the strains studied}

DISCUSSION

It is necessary, before proceeding further, to clarify the relationship of the strains under study. Strains c6, A 20 and s 3 represent separate clonal lines derived from a single isolate from nature, the wild-type IoI. $\mathrm{c} 6$ was isolated as a wild-type clone with stable morphological characteristics. A 20 was selected from a series of cycloheximideresistant mutants (N. Kerr, 1965) and s3 was isolated as a minute mutant which appeared after selection on cycloheximide (N. Kerr, 1967). Variations in chromosome number reported here therefore represent alterations of number within and not between isolates.

\section{Sources of ploidy variation}

Variation in chromosome number of myxomycete nuclei within an amoeboid population or within a single plasmodium is not a new observation. Such variation has been documented recently in plasmodia of Physarum polycephalum (Ross, 1966; Koevenig \& Jackson, 1966) and in amoebae of Badhamia curtisii (Ross, 1966). The variation reported by Ross occurred in strains grown in pure culture whereas variation reported in the present study was found both in 'axenically grown' and bacterialgrown strains.

Phase-contrast observations made on $\mathrm{c} 6$ and s3 living amoebae growing on SS thin agar suggest how ploidy level variation may originate in these strains (S. Kerr, 1968). In brief, fusion of amoebae has been observed in cases where subsequent observations for $24 \mathrm{hr}$ have shown that the resulting cell did not give rise to a plasmodium but continued to divide as an amoeba (class $\mathrm{A}$ to class $\mathrm{C}$ transition). That products of such fusions sometimes do differentiate to plasmodia was demonstrated by isolation of fusing pairs (N. Kerr, 1961). Some cells, generally larger than most in the population, may undergo tripolar divisions (N. Kerr, 1967). Occasionally two of these tripolar daughter cells may fuse. One tripolar metaphase plate was counted and contained in excess of 130 chromosomes. Division of this cell would represent a class D to class $\mathrm{B}$ transition. If a class $\mathrm{C}$ cell divided tripolarly and then two of the daughter cells fused, one of the resulting cells would belong to class B whereas the other would contain less than 30 chromosomes. Fusions and tripolar divisions occur in less than $\mathrm{I} \%$ of the cells of a population if they are detected at all (S. Kerr, 1968). Perhaps it is not unreasonable to suppose that such behaviour could account for variations in chromosome number and that over a period of time the number of cells with chromosome numbers outside the $31-38$ range should increase.

\section{The origin of plasmodia}

The classical myxomycete life-cycle dictates that fusion of gametes, either amoebae or flagellates, serves as an initiating step in the differentiation of the plasmodial stage. Several workers have shown that this is the case in many different organisms, some closely related to the isolate under study. Schünemann (1930) and Cadman (I93I) reported amoeboid fusion in Didymium nigripes. Schünemann followed these fusion products through the stage of the polynuclear young plasmodium and observed no 
karyogamy. Cadman concluded that karyogamy did occur and that subsequently the zygotes fused to form plasmodia.

Three observations exist for the isolate under study. Von Stosch (personal communication to Dr Norman Kerr) concluded that the ancestral ror strain was apogamous, i.e. that meiosis and karyogamy are not present during the life-cycle. His conclusions were based on the absence of meiosis at the time it is found in heterothallic isolates of this species and on chromosome counts which showed approximately the same number of chromosomes in amoeboid and plasmodial nuclei. Von Stosch examined nuclei with chromosome numbers approaching 80. Recently N. Kerr (1967) showed by means of time-lapse cinephotomicrography that the $\mathbf{s} 3$ strain under study is capable of forming plasmodia without either plasmogamy or karyogamy.

Therrien (1966) measured DNA spectrophotometrically in a strain derived from the ancestral wild-type IOI, found two classes of nuclei in his amoeboid cultures, and concluded that the larger cells represented zygotes and that karyogamy preceded plasmodial differentiation. Of 100 amoeboid nuclei he measured, Therrien placed 27 in what he designated as a haploid $2 \mathrm{C}$ class and 73 in a diploid ${ }_{4} \mathrm{C}$ class. In this investigation identical culture conditions were not employed and the only nuclei of strain IOI which were studied were 'axenically' grown amoebae; of these cells $8 \%$ could be placed in a class which corresponds to Therrien's haploid $2 \mathrm{C}$ (class $\mathrm{A}$ ), $8 \%$ in an intermediate class (39-50 chromosomes), $75 \%$ in Therrien's diploid ${ }_{4} \mathrm{C}$ (5I-76 chromosomes) and $6 \%$ in class D. Inspection of Therrien's data shows that he, too, found nuclei with amounts of DNA intermediate to the two ploidy classes and greater than the diploid ${ }_{4} \mathrm{C}$ class. Thus while data from two different techniques agree closely, it is important to note that the 'axenically' grown amoebae used in the present study contain 'diploid' cells which are not plasmodia under liquid culture conditions. When such cells are plated on GPY/5 agar for ' $\mathrm{P}$ ' clone assay (N. Kerr, 196I) only amoeboid plaques are recovered and no ' $\mathrm{P}$ ' clones are formed, indicating that none of the cells plated had undergone plasmodial differentiation before plating even though $75 \%$ of them were presumably 'diploid'. Furthermore, all of the cells plated continued to divide as amoebae for 2 days before plasmodial differentiation began.

Therrien's analysis showed that all the plasmodial nuclei he surveyed belonged to one size class-the diploid ${ }_{4} \mathrm{C}$; it is curious that Therrien chose to recognize only one size class of nuclei in his preparations for examination of his graph suggests that several size classes could have been recognized. Plasmodia of all strains examined in this study, including the wild-type IOI which Therrien used, contained nuclei which differed in size. Examination of 500 nuclei of plasmodia grown from spores stored in lyophil tubes showed, on the basis of a simple nuclear sizing technique (visual estimation of nuclear diameter), that plasmodia derived from spores of the strain given to Therrien contained $78 \%$ large (I $5 \%$ may be polyploid) and $22 \%$ small nuclei.

The present investigation shows that in two strains of the isolate of Didymium nigripes under study there is no ploidy level difference between a population of amoebae and plasmodia derived from this population. Although nuclear and cytoplasmic fusion may precede plasmodial initiation, such fusion is not necessary for plasmodial differentiation to occur. The absence of nuclear fusion prior to plasmodium formation is regarded as evidence supporting the hypothesis that the $\mathrm{c} 6$ and s 3 strains of this isolate may lack a sexual cycle. 


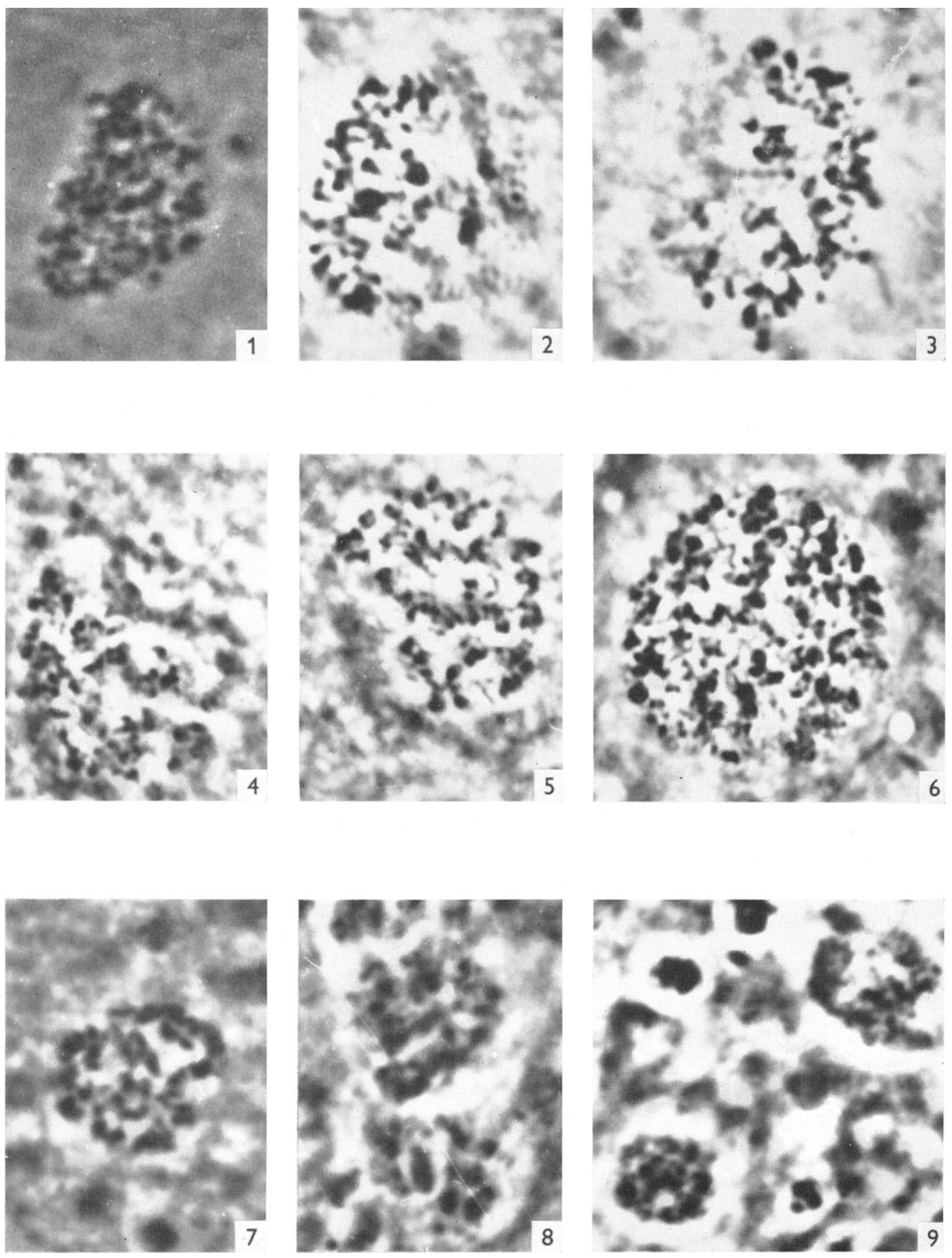
This work was supported partially by NIH, Institute of Allergy and Infectious Disease grant AI-0552I to Dr Norman Kerr and by an American Cancer Society Institutional Research Grant Award made to the author by the Cancer Coordinating Committee of the University of Minnesota.

\section{REFERENCES}

Cadman, E. (193I). The life history and cytology of Didymium nigripes. Trans. r. Soc. Edinb. 57, 93. KERR, N.S. (1960). Flagella formation by myxamoebae of the true slime mold, Didymium nigripes. J. Protozool. 7, 103.

KERR, N. S. (196I). A study of plasmodium formation by the true slime mold, Didymium nigripes. Expl. Cell Res. 23, 603.

KERR, N.S. (1963). The growth of myxamoebae of the true slime mould, Didymium nigripes, in axenic culture. J. gen. Microbiol. 32, 409.

KERR, N. S. (1965). Disappearance of a genetic marker from a cytoplasmic hybrid plasmodium of a true slime mold. Science, N.Y. $\mathbf{x 4 7}, \mathrm{I} 586$.

Kerr, N. S. (1967). Plasmodium formation by a minute mutant of the true slime mold, Didymium nigripes. Expl. Cell Res. 45, 646.

KERR, S. (1967). A comparative study of mitosis in amoebae and plasmodia of the true slime mold, Didymium nigripes. J. Protozool. 14, 439.

KeRr, S. (1968). Cytological observations on plasmodial differentiation in the true slime mold, Didymium nigripes. Ph.D. dissertation, University of Minnesota.

Koevenig, J. L. \& JACKson, R. C. (I966). Plasmodial mitosis and polyploidy in the Myxomycete Physarum polycephalum. Mycologia 53, 662.

Ross, I. K. (1966). Chromosome numbers in pure and gross cultures of myxomycetes. Am.J. Bot. 53, 712.

Schünemann, E. (I930). Untersuchungen über die Sexualität der Myxomycetes. Planta 9, 645.

THERRIEN, C. D. (I966). Microspectrophotometric measurement of nuclear deoxyribonucleic acid content in two myxomycetes. Can. J. Bot. 44, I667.

\section{EXPLANATION OF PLATE}

All figures $\times 4000$.

Fig. I. A20 axenic amoeba; class A.

Fig. 2, 3. Two focal levels through $\mathrm{s}_{3}$ axenic amoeba; Class $\mathrm{C}$.

Fig. 4, 5. Two focal levels through Ior wild-type axenic amoeba; class D.

Fig. 6. 66 plasmodium; prometaphase.

Fig. 7. $\mathrm{c} 6$ axenic amoeba; less than 30 chromosomes.

Fig. 8. C6 axenic plasmodium; class A.

Fig. 9. C6 plasmodium; class A. 